\title{
Technical Report: Voltage Source Converter MPC with Optimized Pulse Patterns and minimization of Integrated Squared Tracking Error
}

\author{
Ivan Pejcic * $^{*} \quad$ Stefan Almér $^{\dagger} \quad$ Helfried Peyrl ${ }^{\dagger}$
}

February 22, 2017

\begin{abstract}
Model predictive control schemes for power electronic applications are characterized by a great variety of problem formulations. In this paper, we consider a three phase voltage source converter with an arbitrary number of voltage levels and derive a model predictive control scheme involving a combination of optimized pulse patterns and the integral of squared predicted tracking error as a cost function. We obtain a nonlinear optimization problem with the switching times as optimization variables, and solve it using gradient projection algorithm. To obtain an easier optimization problem to be solved on-line, a linearization around nominal switching instants is performed bringing the problem to a quadratic programming form. Simulation results demonstrating the performance of the derived scheme are provided for the case of a grid-tied converter with LCL filter.
\end{abstract}

\footnotetext{
*Laboratoire d'Automatique, École Polytechnique Fédérale de Lausanne, 1015 Lausanne, Switzerland, ivan.pejcic@epfl.ch.

${ }^{\dagger}$ ABB Corporate Research, Segelhofstrasse 1K, 5405 Baden-Dättwil, Aargau, Switzerland, \{stefan.almer, helfried.peyrl\}@ch.abb.com.

The research leading to these results has received funding from the European Research Council under the European Union's Seventh Framework Programme (FP/2007-2013) / ERC Grant Agreement n. 307608: BuildNet.
} 


\section{Introduction}

Model predictive control (MPC) schemes developed for power electronic applications have demonstrated improved performance compared to the traditional stateof-the-art control in power electronics [1]. The characteristics of power electronic systems, such as the ability of an inverter to supply a finite number of voltage levels and very limited computational power within the sampling intervals, have caused the power electronic MPC schemes to be highly tailored to the application and to be quite different from the standard MPC formulations studied in the control community.

The aforementioned MPC power electronic schemes thus involve a great variety of "problem-tailored" cost functions. For example, the MPC in [2] involves a cost which minimizes the converter switching frequency in order to indirectly reduce the commutation losses of the power converter. Another MPC approach for power electronics [3], involves a cost function which employs superposition of correction pulses to eliminate an error vector over the prediction horizon. In [4], [5], the cost function is formulated so that it directly addresses the commutation losses of a power converter.

The goal of this paper is to develop a controller using the integral square tracking error as a cost function together with optimized pulse patterns (OPPs). The OPPs are precomputed converter voltage waveforms optimized for high steadystate performance with a fixed number of converter's switchings [6], [7]. Integral squared problem formulations for power electronic applications were previously considered in $[8,9,10]$ in combination with so-called fixed frequency pulse-width modulation (PWM). The use of OPPs in this paper instead of PWM allows to further reduce the harmonic distortion caused by the converter. In comparison to [3] which provides an MPC scheme as well employing OPPs, our approach involves a cost function that penalizes the squared tracking error along the prediction horizon and is in addition applicable to a greater variety of power electronic systems, though at the expense of more demanding computations.

The development considers the power electronics system in state-space representation and thus covers a wide variety of power electronic configurations. Nevertheless, to facilitate an easier exposition we consider a particular setting common in power electronic applications, which involves a power converter connected to the power grid via an $L C L$ filter. By employing OPPs, the discrete decision variables coming from the finite number of converter voltage levels will get eliminated, and the development results in a nonlinear optimization problem with only switching times as continuous decision variables. The optimization problem is solved by applying gradient projection (GP) algorithm operated based on cost function descent. To allow a potentially more convenient optimization problem for an on-line application, a linearization around the OPP switching times is per- 
formed which brings the problem to the form of quadratic programming (QP), with decision variables being the deviations from the OPP (nominal) switching times.

The structure of the paper is as follows. Section 2 describes a model of the system. Section 3 formulates the control optimization problem, and section 4 describes the GP and QP solution approach to it. Section 5 provides the simulation results demonstrating the performance of the method. Section 6 provides conclusion.

\section{System Model}

The configuration with power converter interconnected to the power grid via an $L C L$ filter is presented on Fig. 1. The model of the system consists of the $L C L$ filter, power grid, and the power converter.

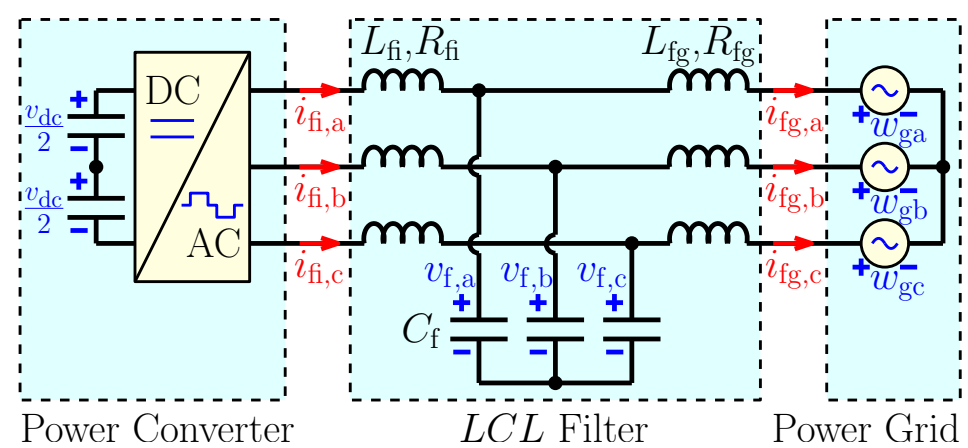

Figure 1: The configuration with a power converter connected to the power grid via an $L C L$ filter.

\section{1 $L C L$ filter}

The state of the $L C L$ filter is described in stationary abc frame with state vector

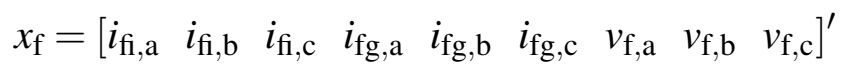

where for each phase $p \in\{\mathrm{a}, \mathrm{b}, \mathrm{c}\}, i_{\mathrm{fi}, \mathrm{p}}, i_{\mathrm{fg}, \mathrm{p}}$ and $v_{\mathrm{f}, \mathrm{p}}$ denote the inverter current, grid current and capacitor voltage, respectively. The $L C L$ filter dynamics are

$$
\dot{x}_{\mathrm{f}}(t)=A_{\mathrm{f}} x_{\mathrm{f}}(t)+B_{\mathrm{f}} s(t)+F_{\mathrm{f}} w_{\mathrm{g}}(t)
$$

where $s=\left[s_{\mathrm{a}}, s_{\mathrm{b}}, s_{\mathrm{c}}\right]^{\prime} \in \mathbb{R}^{3}$ is the vector of converter switch signals (further modeled in Section 2.4) and $w_{\mathrm{g}}=\left[w_{\mathrm{ga}}, w_{\mathrm{gb}}, w_{\mathrm{gc}}\right]^{\prime} \in \mathbb{R}^{3}$ is a vector representing the 
grid voltage (further modeled in Section 2.2). The system matrices are obtained by applying Kirchhoff's circuit laws and have the form

$$
\begin{aligned}
A_{\mathrm{f}}= & {\left[\begin{array}{ccc}
-\frac{R_{\mathrm{fi}}}{L_{\mathrm{fi}}} \mathrm{I}_{3 \times 3} & 0_{3 \times 3} & -\frac{1}{L_{\mathrm{fi}}} \mathrm{I}_{3 \times 3} \\
0_{3 \times 3} & -\frac{R_{\mathrm{fg}}}{L_{\mathrm{fg}}} \mathrm{I}_{3 \times 3} & \frac{1}{L_{\mathrm{fg}}} \mathrm{I}_{3 \times 3} \\
\frac{1}{C_{\mathrm{f}}} \mathrm{I}_{3 \times 3} & -\frac{1}{C_{\mathrm{f}}} \mathrm{I}_{3 \times 3} & 0_{3 \times 3}
\end{array}\right], } \\
B_{\mathrm{f}}= & \frac{1}{L_{\mathrm{fi}}} \frac{1}{3}\left[\begin{array}{ccc}
2 & -1 & -1 \\
-1 & 2 & -1 \\
-1 & -1 & 2 \\
0_{6 \times 1} & 0_{6 \times 1} & 0_{6 \times 1}
\end{array}\right] \frac{v_{\mathrm{dc}}}{2}, \quad F_{\mathrm{f}}=\left[\begin{array}{c}
0_{3 \times 3} \\
-\frac{1}{L_{\mathrm{fg}}} \mathrm{I}_{3 \times 3} \\
0_{3 \times 3}
\end{array}\right],
\end{aligned}
$$

where the parameters correspond to the elements as illustrated in Fig. 1, and $\mathrm{I}_{m \times m}$ and $0_{m \times n}$ are the identity and zero matrix of specified dimensions, respectively. The DC side capacitor voltage $v_{\mathrm{dc}}$ is assumed constant and is "absorbed" into the affine term $B_{\mathrm{f}}$ of the dynamics.

\subsection{Grid voltage}

The three-phase grid voltage $w_{\mathrm{g}}=\left[w_{\mathrm{ga}}, w_{\mathrm{gb}}, w_{\mathrm{gc}}\right]^{\prime}$ is assumed (I) sinusoidal and (II) three-phase symmetric:

$$
\begin{aligned}
\text { (I) } w_{\mathrm{ga}}(t) & =V_{\mathrm{g}} \sin \left(2 \pi f_{\mathrm{g}} t+\varphi_{\mathrm{g}}\right), \\
\text { (II) } w_{\mathrm{ga}}(t) & =w_{\mathrm{gb}}\left(t-1 /\left(3 f_{\mathrm{g}}\right)\right)=w_{\mathrm{gc}}\left(t+1 /\left(3 f_{\mathrm{g}}\right)\right),
\end{aligned}
$$

where $V_{\mathrm{g}}$ is the voltage amplitude, $f_{\mathrm{g}}$ is the frequency and $\varphi_{\mathrm{g}}$ is the initial phase of the grid. An alternative possibility is to model the grid voltage as being constant over the prediction horizon.

The sinusoidal three-phase symmetric voltage can be modeled in $\alpha \beta$ coordiante system $w_{\mathrm{g} \alpha \beta}(t)=\left[w_{\mathrm{g} \alpha}(t), w_{\mathrm{g} \beta}(t)\right]^{\prime}$ with

$$
\dot{w}_{\mathrm{g} \alpha \beta}(t)=R_{2 \times 2} w_{\mathrm{g} \alpha \beta}(t), \quad R_{2 \times 2}:=\left[\begin{array}{cc}
0 & -\omega_{g} \\
\omega_{g} & 0
\end{array}\right],
$$

where $\omega_{\mathrm{g}}=2 \pi f_{\mathrm{g}}$ is the grid angular frequency. The three-phase voltage $w_{\mathrm{g}}(t)$ is then obtained from $w_{\mathrm{g} \alpha \beta}(t)$ using

$$
w_{\mathrm{g}}(t)=P_{3 \times 2}^{-1} w_{\mathrm{g} \alpha \beta}(t), \quad P_{3 \times 2}^{-1}:=\left[\begin{array}{cc}
1 & 0 \\
-\frac{1}{2} & \frac{\sqrt{3}}{2} \\
-\frac{1}{2} & -\frac{\sqrt{3}}{2}
\end{array}\right],
$$

where $P_{3 \times 2}^{-1}$ denotes the inverse Clarke transform [11]. 


\subsection{State-space model}

The state-space model is obtained by combining the $L C L$ dynamics (2) and the grid voltage model (5), taking the form

$$
\dot{x}_{\mathrm{s}}(t)=A_{\mathrm{s}} x_{\mathrm{s}}(t)+B_{\mathrm{s}} s(t)
$$

where

$$
x_{\mathrm{s}}=\left[\begin{array}{c}
x_{\mathrm{f}} \\
w_{\mathrm{g} \alpha \beta}
\end{array}\right], A_{\mathrm{s}}=\left[\begin{array}{cc}
A_{\mathrm{f}} & F_{\mathrm{f}} P_{3 \times 2}^{-1} \\
0_{2 \times 9} & R_{2 \times 2}
\end{array}\right], B_{\mathrm{s}}=\left[\begin{array}{c}
B_{\mathrm{f}} \\
0_{2 \times 3}
\end{array}\right] .
$$

\subsection{Voltage source converter}

The power converter [12] is modeled over the prediction horizon by the sequence of voltage levels which it provides. The naming conventions introduced in the sequel are illustrated with an example in Fig. 2. We assume the components $s_{i}(t), i \in\{\mathrm{a}, \mathrm{b}, \mathrm{c}\}$, of the switch vector $s(t)$ take $N_{\mathrm{lvl}}$ distinct values

$$
s_{i} \in\left\{v_{1}, v_{2}, \ldots, v_{N_{\mathrm{lvl}}}\right\},
$$

which correspond to the voltage levels the power converter can suply. In the MPC problem we consider a prediction over the window $\left[t_{0}, t_{1}\right], t_{0}<t_{1}$, and within it we allow the total number of switches in all three phases be $N_{\mathrm{sw}}$. Thus, $N_{\mathrm{sw}}=N_{\mathrm{a}}+$ $N_{\mathrm{b}}+N_{\mathrm{c}}$ where $N_{i}, i \in\{\mathrm{a}, \mathrm{b}, \mathrm{c}\}$ is the number of switchings in phase $i$. The switch signal $s_{i}(t)$ takes $N_{i}+1$ different values within the prediction window $\left[t_{0}, t_{1}\right]$.

The transition time of the $j^{\text {th }}$ switch in phase $i$ is denoted $t_{i j}$ where $j \in\left\{1, \ldots, N_{i}\right\}$, $i \in\{\mathrm{a}, \mathrm{b}, \mathrm{c}\}$, and the convention is $t_{i j} \leq t_{i j+1}$. The voltage level applied during the time between $t_{i j}$ and $t_{i j+1}$ is denoted $\ell_{i j+1}$. Thus, the switch signals can be written on the form

$$
s_{i}(t)= \begin{cases}\ell_{i 1}, & t \in\left[t_{0}, t_{i 1}\right) \\ \ell_{i 2}, & t \in\left[t_{i 1}, t_{i 2}\right) \\ \vdots & \vdots \\ \ell_{i N_{i},} & t \in\left[t_{i N_{i}-1}, t_{i N_{i}}\right) \\ \ell_{i N_{i}+1}, & t \in\left[t_{i N_{i}}, t_{1}\right]\end{cases}
$$

where $\ell_{i j} \in\left\{v_{1}, v_{2}, \ldots, v_{N_{\mathrm{lvl}}}\right\}$.

\section{Cost Function and Constraint Set}

The cost function comprises an integral of squared difference between a reference signal and predicted trajectory. The reference signals corresponding to desired 


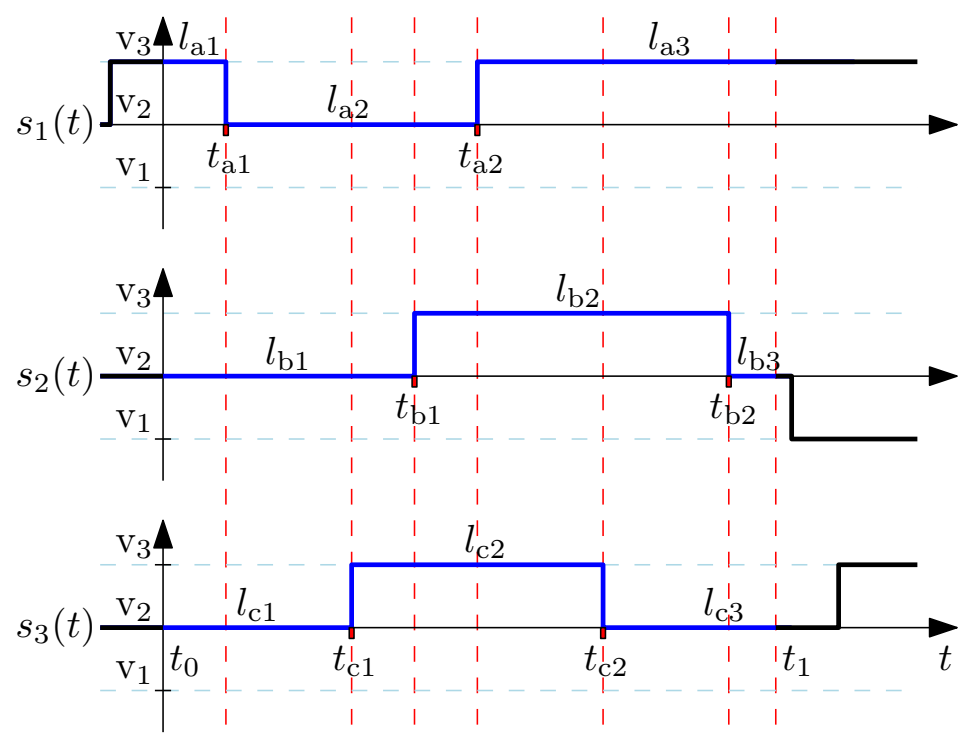

Figure 2: An example of three-phase switch signal $s(t)$, showing switching times and discrete amplitudes. The example involves $N_{\mathrm{lvl}}=3$ and $N_{\mathrm{sw}}=6$.

active and reactive power injections to the grid are approximated by sinusoidal shapes. The discrete-valued voltage levels are ruled out from decision variables by using OPPs.

\subsection{Sinusoidal steady-state reference}

The steady-state reference for each state of the $L C L$ filter (1) is approximated by a sinusoidal shape. Assuming a three-phase symmetric and sinusoidal steady-state, the reference vector $x_{\mathrm{r}} \in \mathbb{R}^{9}$ can be modeled in $\alpha \beta$ coordinate frame by

$$
\dot{x}_{\mathrm{r} \alpha \beta}(t)=R_{6 \times 6} x_{\mathrm{r} \alpha \beta}(t), \quad R_{6 \times 6}:=\operatorname{blkdiag}\left(R_{2 \times 2}, R_{2 \times 2}, R_{2 \times 2}\right),
$$

where

$$
x_{\mathrm{r} \alpha \beta}=\left[\begin{array}{llllll}
i_{\mathrm{r}, \mathrm{fi} \alpha} & i_{\mathrm{r}, \mathrm{fi} \beta} & i_{\mathrm{r}, \mathrm{fg} \alpha} & i_{\mathrm{r}, \mathrm{fg} \beta} & v_{\mathrm{r}, \mathrm{f} \alpha} & v_{\mathrm{r}, \mathrm{f} \beta}
\end{array}\right]^{\prime}
$$

consists of $L C L$ state references in $\alpha \beta$ frame, and the $R_{2 \times 2}$ is the matrix defined in (5). To convert the references from $\alpha \beta$ to abc coordinate frame, one employs the inverse Clarke transform (6) as

$$
x_{\mathrm{r}}(t)=P_{9 \times 6}^{-1} x_{\mathrm{r} \alpha \beta}(t), P_{9 \times 6}^{-1}:=\operatorname{blkdiag}\left(P_{3 \times 2}^{-1}, P_{3 \times 2}^{-1}, P_{3 \times 2}^{-1}\right) .
$$

The initial values $x_{\mathrm{r} \alpha \beta}\left(t_{0}\right)$ of the model in $\alpha \beta$ frame (10) at the beginning of the prediction horizon $t_{0}$ are determined from the specified active $P_{\mathrm{g}}$ and reactive 
$Q_{\mathrm{g}}$ power to be injected to the grid. These powers are equal to

$$
\begin{aligned}
& P_{\mathrm{g}}=3 V_{\mathrm{g}} I_{\mathrm{fg}} \cos \left(\theta_{\mathrm{g}}\right), \\
& Q_{\mathrm{g}}=3 V_{\mathrm{g}} I_{\mathrm{fg}} \sin \left(\theta_{\mathrm{g}}\right),
\end{aligned}
$$

where $V_{\mathrm{g}}$ is the grid voltage amplitude as in (3), $I_{\mathrm{fg}}$ is the grid current amplitude, and $\theta_{\mathrm{g}}$ is the phase lag between the grid voltage and current. This yields the desired steady-state amplitude $I_{\text {fg }}$ and phase lag $\theta_{\mathrm{g}}$ :

$$
I_{\mathrm{fg}}=\frac{1}{3 V_{\mathrm{g}}} \sqrt{P_{\mathrm{g}}^{2}+Q_{\mathrm{g}}^{2}}, \quad \theta_{\mathrm{g}}=\arctan \left(\frac{Q_{\mathrm{g}}}{P_{\mathrm{g}}}\right) .
$$

Introducing a phasor $\underline{\mathrm{V}}_{\mathrm{g}}=w_{\mathrm{g} \alpha}\left(t_{0}\right)+\mathrm{jw}_{\mathrm{g} \beta}\left(\mathrm{t}_{0}\right)$ where $\mathrm{j}=\sqrt{-1}$, the phasor modeling the desired grid current is

$$
\underline{\mathrm{I}}_{\mathrm{fg}}=I_{\mathrm{fg}} e^{\mathrm{j}\left\{\arg \left(\underline{\mathrm{V}}_{\mathrm{g}}\right)-\theta_{\mathrm{g}}\right\}} .
$$

Using this value, the phasors of the desired capacitor voltage and inverter current are

$$
\underline{\mathrm{V}}_{\mathrm{f}}=\underline{\mathrm{V}}_{\mathrm{g}}+\mathrm{j} \omega_{\mathrm{g}} \mathrm{L}_{\mathrm{fg}} \underline{\mathrm{I}}_{\mathrm{g}}, \quad \underline{\mathrm{I}}_{\mathrm{fi}}=\mathrm{j} \omega_{\mathrm{g}} \mathrm{C}_{\mathrm{f}} \underline{\mathrm{V}}_{\mathrm{f}}+\underline{\mathrm{I}}_{\mathrm{fg}} .
$$

The desired initial conditions of the $x_{r \alpha \beta}$ at the beginning of the prediction horizon (time $t_{0}$ ) are thus

$$
\begin{aligned}
i_{\mathrm{r}, \mathrm{fi} \alpha}\left(t_{0}\right) & =\operatorname{Re}\left\{\underline{\mathrm{I}}_{\mathrm{fi}}\right\}, & & i_{\mathrm{r}, \mathrm{f} \beta}\left(t_{0}\right)=\operatorname{Im}\left\{\underline{\mathrm{I}}_{\mathrm{f}}\right\}, \\
i_{\mathrm{r}, \mathrm{fg} \alpha}\left(t_{0}\right) & =\operatorname{Re}\left\{\underline{\mathrm{I}}_{\mathrm{fg}}\right\}, & & i_{\mathrm{r}, \mathrm{fg} \beta}\left(t_{0}\right)=\operatorname{Im}\left\{\underline{\mathrm{I}}_{\mathrm{fg}}\right\}, \\
v_{\mathrm{r}, \mathrm{f} \alpha}\left(t_{0}\right) & =\operatorname{Re}\left\{\underline{\mathrm{V}}_{\mathrm{f}}\right\}, & & v_{\mathrm{r}, \mathrm{f} \beta}\left(t_{0}\right)=\operatorname{Im}\left\{\underline{\mathrm{V}}_{\mathrm{f}}\right\} .
\end{aligned}
$$

The desired sinusoidal inverter voltage which leads to the desired steady-state is

$$
\underline{\mathrm{V}}_{\mathrm{i}}=\underline{\mathrm{V}}_{\mathrm{f}}+\mathrm{j} \omega_{\mathrm{g}} \mathrm{L}_{\mathrm{fi}} \underline{\mathrm{I}}_{\mathrm{f}} \text {. }
$$

This voltage will correspond to the fundamental harmonic of the OPP employed by the converter, as described in the section about OPPs in the sequel.

There are several aspects that support the approximation of references by sinusoids. It turns out that the actual deviations from sinusoidal shapes in steadystate seem significant only for the inverter current, while for the grid current and capacitor voltage the deviations are hardly visually noticeable. In case of $L C L$ filter parameters which cause more significant deviations from sinusoids, one may introduce into the reference some higher harmonics, which are computable from the corresponding higher harmonics of utilised OPP. Furthermore, the small steady-state deviations from the ideal sinusoidal shapes do not seem capable to cause perturbations larger than the errors coming form the inevitable real-world non-idealities of the system such as variations of the DC link voltage, temperature-dependent resistances, inverter's non-idealities like dead-time, and state-estimation errors. 


\subsection{Augmented state-space model}

To take the model of sinusoidal references (10)-(12) into account, we augment the state-space model (7) and obtain

$$
\dot{x}(t)=A x(t)+B s(t)
$$

where

$$
x=\left[\begin{array}{c}
x_{\mathrm{s}} \\
x_{\mathrm{r} \alpha \beta}
\end{array}\right], \quad A=\left[\begin{array}{cc}
A_{\mathrm{s}} & 0_{11 \times 6} \\
0_{6 \times 11} & R_{6 \times 6}
\end{array}\right], \quad B=\left[\begin{array}{c}
B_{\mathrm{S}} \\
0_{6 \times 3}
\end{array}\right] .
$$

Since the goal is to minimize the integral of the squared tracking error, the output is selected to be the deviation of state from the reference trajectory:

$$
y(t)=E x(t), \quad E=\left[\begin{array}{lll}
I_{9 \times 9} & 0_{9 \times 2} & -P_{9 \times 6}^{-1}
\end{array}\right] .
$$

\subsection{Optimized pulse patterns}

As described in Section 2.4, the switch signal $s(t) \in \mathbb{R}^{3}$ depends on switching times $t_{i j}$ (continuous decision variables) and voltage levels $\ell_{i j}$ (discrete decision variables). To avoid optimization over the voltage levels, we will copy them from an off-line computed steady-state waveform (the OPP) whose fundamental voltage harmonic (the amplitude and phase) corresponds to the desired sinusoidal steady-state voltage, which is the $\underline{\mathrm{V}}_{\mathrm{i}}$ from (21).

The OPPs [7], [3] are steady-state converter waveforms obtained by off-line optimization for a specified number of switchings within the period $1 / f_{\mathrm{g}}$ and fundamental voltage harmonic $\mathrm{V}_{\mathrm{i}}$. The optimization minimizes a total harmonic distortion (THD) of grid current, the steady-state performance quantifier defined as

$$
\operatorname{THD}\left(\mathrm{I}_{\mathrm{fg}}\right)=\frac{\sqrt{\sum_{\mathrm{k}=2}^{\infty} \mathrm{I}_{\mathrm{fg}, \mathrm{k}}^{2}}}{\mathrm{I}_{\mathrm{fg}, 1}},
$$

where the $I_{\mathrm{fg}, \mathrm{k}}$ represents the k-th harmonic of the grid current in one of the phases. The OPPs are commonly computed by assuming a quarter-wave symmetry, in which case the number of switchings in the first quarter of the period is the same as the number of the switchings in each of the other quarters and is called a pulse number. To be able to select an OPP which has the fundamental component conveniently close to the desired sinusoid, the OPPs are calculated by gridding an interval of magnitudes $\left[V_{i, \min }, V_{\mathrm{i}, \max }\right]$ which covers the operating range of the converter. 


\subsection{Predicted state trajectory}

To explicitly formulate the solution of the system (23) we need to fix the order of the transitions of the switches of each phase with respect to one another. The single phase voltage levels of $s(t)$ are obtained from an OPP, and we also need to determine what voltage levels are applied to the three phases in between transition times. The sequence of three-phase voltage levels is determined by the sequence of single phase voltage levels $\ell_{i j}, i \in\{\mathrm{a}, \mathrm{b}, \mathrm{c}\}$ and the sequence of switching times. For example, we may consider the switching order illustrated in Fig. 2 where $N_{\text {sw }}=6$ and

$$
t_{0} \leq t_{\mathrm{a} 1} \leq t_{\mathrm{c} 1} \leq t_{\mathrm{b} 1} \leq t_{\mathrm{a} 2} \leq t_{\mathrm{c} 2} \leq t_{\mathrm{b} 2} \leq t_{1}
$$

The corresponding sequence of $N_{\mathrm{sw}}+1$ three-phase voltage levels is

$$
\left[\begin{array}{l}
\ell_{a 1} \\
\ell_{b 1} \\
\ell_{c 1}
\end{array}\right],\left[\begin{array}{l}
\ell_{a 2} \\
\ell_{b 1} \\
\ell_{c 1}
\end{array}\right],\left[\begin{array}{l}
\ell_{a 2} \\
\ell_{b 1} \\
\ell_{c 2}
\end{array}\right],\left[\begin{array}{l}
\ell_{a 2} \\
\ell_{b 2} \\
\ell_{c 2}
\end{array}\right],\left[\begin{array}{l}
\ell_{a 3} \\
\ell_{b 2} \\
\ell_{c 2}
\end{array}\right],\left[\begin{array}{l}
\ell_{a 3} \\
\ell_{b 2} \\
\ell_{c 3}
\end{array}\right],\left[\begin{array}{l}
\ell_{a 3} \\
\ell_{b 3} \\
\ell_{c 3}
\end{array}\right] .
$$

We now proceed to introduce index vectors which represent the order of the switching times and corresponding three-phase voltage levels. To describe the transition times we introduce the index vector $\bar{I}$ which is a vector of dimension $N_{\mathrm{sw}}$ containing the indexes $i, j$ of the switch transitions in a given order:

$$
\bar{I}=[i j], \quad i \in\{\mathrm{a}, \mathrm{b}, \mathrm{c}\}, \mathrm{j} \in\left\{1, \ldots, \mathrm{N}_{\mathrm{i}}\right\} .
$$

For example, the index vector $\bar{I}$ corresponding to (26) is

$$
\bar{I}=\left[\begin{array}{llllll}
\mathrm{a} 1 & \mathrm{c} 1 & \mathrm{~b} 1 & \mathrm{a} 2 & \mathrm{c} 2 & \mathrm{~b} 2
\end{array}\right]^{\prime} .
$$

To handle the boundary conditions with compact expressions we augment the index vector $\bar{I}$ with zero and one and define

$$
I:=\left[\begin{array}{lll}
0 & \bar{I}^{\prime} & 1
\end{array}\right]^{\prime} .
$$

The augmented index vector $I$ has dimension $N_{\mathrm{sw}}+2$. We let the index denoting the entries of $I$ start with zero so that

$$
I_{k}= \begin{cases}0, & k=0 \\ i j, & k \in\left\{1, \ldots, N_{\mathrm{sw}}\right\} \\ 1, & k=N_{s w}+1\end{cases}
$$

The index set $I$ defines the sorted vector of switching times

$$
t_{I}=\left[\begin{array}{lllllll}
t_{0} & t_{I_{1}} & t_{I_{2}} & \ldots & t_{I_{N_{s w}-1}} & t_{I_{N_{S w}}} & t_{1}
\end{array}\right]^{\prime} .
$$


To describe the three-phase voltage levels we introduce the index matrix $J$ defined as

$$
J=\left[J_{0}, \ldots, J_{N_{\mathrm{sw}}}\right]
$$

where

$$
J_{0}=\left[\begin{array}{lll}
a 1 & b 1 & c 1
\end{array}\right]^{\prime}
$$

and the remaining columns are determined by treating the indices $a, b, c$ as values $1,2,3$, respectively, and using the relation

$$
J_{k}=J_{k-1}+e_{\left(I_{k}\right)_{1}} \cdot(01), \quad k=1, \ldots, N_{\mathrm{sw}}
$$

where $e_{j} \in \mathbb{R}^{3}$ is the $j^{\text {th }}$ unit vector and where $\left(I_{k}\right)_{1}$ denotes the first part (the " $i$ ") of the $k^{\text {th }}$ entry of $I$. For example, the index matrix $J$ corresponding to (27) is

$$
J=\left[\left[\begin{array}{l}
a 1 \\
b 1 \\
c 1
\end{array}\right],\left[\begin{array}{l}
a 2 \\
b 1 \\
c 1
\end{array}\right],\left[\begin{array}{l}
a 2 \\
b 1 \\
c 2
\end{array}\right],\left[\begin{array}{l}
a 2 \\
b 2 \\
c 2
\end{array}\right],\left[\begin{array}{l}
a 3 \\
b 2 \\
c 2
\end{array}\right],\left[\begin{array}{l}
a 3 \\
b 2 \\
c 3
\end{array}\right],\left[\begin{array}{l}
a 3 \\
b 3 \\
c 3
\end{array}\right]\right]
$$

The index vector $J_{k}$ is mapped to the three-phase voltage levels by

$$
\mathscr{L}_{k}=\mathscr{L}_{k}\left(J_{k}\right)=\left[\begin{array}{lll}
\ell_{\left(J_{k}\right)_{1}} & \ell_{\left(J_{k}\right)_{2}} & \ell_{\left(J_{k}\right)_{3}}
\end{array}\right]^{\prime}
$$

where $\left(J_{k}\right)_{i}$ denotes the $i^{\text {th }}$ entry of $J_{k}$.

We are now ready to derive an expression for the system solution. Given a fixed switching order $I$, we split the prediction window into $N_{\mathrm{sw}}+1$ subintervals according to

$$
\left[t_{0}, t_{1}\right]=\left[t_{0}, t_{I_{1}}\right) \cup\left[t_{I_{1}}, t_{I_{2}}\right) \cup \cdots \cup\left[t_{I_{N_{\mathrm{sw}}-1}}, t_{I_{N_{\mathrm{sw}}}}\right) \cup\left[t_{I_{N_{\mathrm{sw}}}}, t_{1}\right] .
$$

We proceed to derive an explicit expression for the (continuous time) state over the subinterval $\left[t_{I_{k}}, t_{I_{k+1}}\right)$. Define

$$
x_{k}:=x\left(t_{I_{k}}\right), k=0, \ldots, N_{\mathrm{sw}}+1 .
$$

Using the equality

$$
e^{A p} x+\int_{0}^{p} e^{A(p-\tau)} B \mathrm{~d} \tau=\left[\begin{array}{ll}
I_{n \times n} & 0_{n \times 1}
\end{array}\right] \mathrm{e}^{\left[\begin{array}{cc}
A & B \\
0_{1 \times n} & 0_{1 \times 1}
\end{array}\right] \mathrm{p}}\left[\begin{array}{c}
x \\
1
\end{array}\right]
$$


where $n$ is the dimension of the square matrix $A$, the solution of (23) over the subinterval $\left[t_{I_{k}}, t_{I_{k+1}}\right)$ can be written as

$$
\begin{aligned}
x(t) & =e^{A\left(t-t_{I_{k}}\right)} x_{k}+\int_{t_{I_{k}}}^{t} e^{A(t-\tau)} B \mathscr{L}_{k} \mathrm{~d} \tau \\
& =C e^{\bar{A}_{k}\left(t-t_{I_{k}}\right)} \bar{x}_{k}, \quad t \in\left[t_{I_{k}}, t_{I_{k+1}}\right), k=0, \ldots, N_{\mathrm{sw}},
\end{aligned}
$$

where we have introduced the definitions

$$
C:=\left[\begin{array}{ll}
I_{n \times n} & 0_{n \times 1}
\end{array}\right], \quad \bar{A}_{k}:=\left[\begin{array}{cc}
A & B \mathscr{L}_{k} \\
0_{1 \times n} & 0_{1 \times 1}
\end{array}\right], \quad \bar{x}_{k}:=\left[\begin{array}{c}
x_{k} \\
1
\end{array}\right] .
$$

The solution (29) can be written in a more compact form by eliminating the state variables $x_{k}$. It holds

$$
x(t)=C e^{\bar{A}_{k}\left(t-t_{I_{k}}\right)} \prod_{m=0}^{k-1} e^{\bar{A}_{m}\left(t_{I_{m+1}}-t_{I_{m}}\right)} \bar{x}_{0}
$$

where $t \in\left[t_{I_{k}}, t_{I_{k+1}}\right), k=0, \ldots, N_{\mathrm{sw}}$.

\subsection{Integral of squared predicted tracking error}

We now formulate the cost representing the integral of the squared predicted deviation between $L C L$ states $x_{\mathrm{f}}(t)$ and the references $x_{r}(t)$ over the prediction horizon $\left[t_{0}, t_{1}\right]$ :

$$
\begin{aligned}
J_{\mathrm{c}} & =\int_{t_{0}}^{t_{1}} y(t)^{\prime} Q y(t) \mathrm{dt}=\sum_{\mathrm{k}=0}^{\mathrm{N}_{\mathrm{sw}}} \int_{\mathrm{t}_{\mathrm{k}}}^{\mathrm{t}_{\mathrm{k}+1}} \mathrm{y}(\mathrm{t})^{\prime} \mathrm{Qy}(\mathrm{t}) \mathrm{dt} \\
& =\sum_{k=0}^{N_{S w}} \int_{t_{I_{k}}}^{t_{I_{k+1}}}(E x(t))^{\prime} Q(E x(t)) \mathrm{dt} \\
& =\sum_{k=0}^{N_{S w}} \bar{x}_{k}^{\prime}\left(\int_{t_{I_{k}}}^{t_{I_{k+1}}} e^{\bar{A}_{k}^{\prime}\left(t-t_{I_{k}}\right)}\left(C^{\prime} E^{\prime} Q C E\right) e^{\bar{A}_{k}\left(t-t_{I_{k}}\right)} \mathrm{dt}\right) \overline{\mathrm{x}}_{\mathrm{k}} .
\end{aligned}
$$

Using the results of [13], the cost function becomes

$$
J_{\mathrm{c}}=\sum_{k=0}^{N_{s w}} \bar{x}_{k}^{\prime} N_{k} \bar{x}_{k}
$$


where

$$
N_{k}=F_{k 3}^{\prime} G_{k 2}, \quad k=0, \ldots, N s w,
$$

with $F_{k 3}$ and $G_{k 2}$ obtained by first computing

$$
\hat{A}_{k}=\left[\begin{array}{cc}
-\bar{A}_{k}^{\prime} & C^{\prime} E^{\prime} Q E C \\
0_{(n+1) \times(n+1)} & \bar{A}_{k}
\end{array}\right]
$$

and then taking $F_{k 3}$ and $G_{k 2}$ as submatrices of

$$
e^{\hat{A}_{k}\left(t_{I_{k+1}}-t_{I_{k}}\right)}=\left[\begin{array}{cc}
F_{k 2} & G_{k 2} \\
0_{(n+1) \times(n+1)} & F_{k 3}
\end{array}\right] .
$$

\subsection{Cost function}

The cost function depends on the continuous decision variables $t_{i j}$ within the prediction horizon $\left[t_{0}, t_{1}\right]$ denoted as

$$
\bar{t}=\left[\begin{array}{lllllllll}
t_{\mathrm{a} 1} & \ldots & t_{\mathrm{aN}} & t_{\mathrm{b} 1} & \ldots & t_{\mathrm{bN}_{\mathrm{b}}} & t_{\mathrm{c} 1} & \ldots & t_{\mathrm{cN}_{\mathrm{c}}}
\end{array}\right]^{\prime} .
$$

The times $t_{i j}$ are selected so that they correspond to the nominal switching times of the OPP. The cost has the form

$$
J_{\text {tot }}(\bar{t})=J_{\mathrm{c}}(\bar{t})+J_{\mathrm{d}}(\bar{t})
$$

where $J_{\mathrm{c}}$ is the integrated squared tracking error from (33), and $J_{\mathrm{d}}$ is a quadratic penalty on deviations of switching times $t_{i j}$ from their corresponding nominal switching times $t_{i j}^{*}$ of the off-line computed OPP:

$$
J_{\mathrm{d}}=\sum_{i=1}^{3} \sum_{j=1}^{N} q\left(t_{i j}-t_{i j}^{*}\right)^{2},
$$

with the values $i=1,2,3$ corresponding to the phases a, b,c, respectively, and $q \geq 0$. It should be noted that evaluation of the cost term $J_{\mathrm{c}}$ requires sorting of the vector $\bar{t}$ with switching times to the vector $t_{I}$ with monotonically non-decreasing times, as described in Section 3.4.

\subsection{Constraint set}

The constraint involved in the optimization problem imposes the fixed order of the switching times in each of the three phases idependently:

$$
\begin{array}{cccc}
t_{0} \leq t_{\mathrm{a} 1}, & t_{\mathrm{a} 1}+\delta \leq t_{\mathrm{a} 2}, \quad \ldots, & t_{\mathrm{aN}}+\delta \leq t_{1}, \\
t_{0} \leq t_{\mathrm{b} 1}, & t_{\mathrm{b} 1}+\delta \leq t_{\mathrm{b} 2}, \ldots, & t_{\mathrm{bN}_{\mathrm{b}}}+\delta \leq t_{1}, \\
t_{0} \leq t_{\mathrm{c} 1}, & t_{\mathrm{c} 1}+\delta \leq t_{\mathrm{c} 2}, \ldots, & t_{\mathrm{cN}_{\mathrm{c}}}+\delta \leq t_{1},
\end{array}
$$

where $\delta$ is the minimal allowed separation between any two consecutive switching times in each phase, except between $t_{0}$ and $t_{i 1}, i \in\{\mathrm{a}, \mathrm{b}, \mathrm{c}\}$. 


\section{Solution Approaches}

The control optimization problem consisting of the cost (38) and the constraint set (40)-(42) is solved in two ways. The first applies GP algorithm, and the second performs linearization of the state vectors $\bar{x}_{k}$ to obtain a QP form.

\subsection{Gradient projection}

By denoting with $X$ the poytopic constraint set defined by (40)-(42), the GP algorithm [14] takes the form

$$
\bar{t}_{k+1}=P_{X}\left(\bar{t}_{k}-s_{\mathrm{gp}, \mathrm{k}} \nabla J_{t o t}\left(\bar{t}_{k}\right)\right),
$$

where $\bar{t}_{k} \in \mathbb{R}^{N_{s w}}$ is the vector of switching times as in (37) at $k$-th iteration of gradient projection algorithm, $P_{X}: \mathbb{R}^{N_{s w}} \rightarrow \mathbb{R}^{N_{s w}}$ denotes the projection on the set $X \subset \mathbb{R}^{N_{s w}}, s_{\mathrm{gp}, \mathrm{k}}>0$ is the stepsize at the iteration $k$ chosen so that there holds

$$
J\left(\bar{t}_{k}\right)-J\left(\bar{t}_{k+1}\right) \geq \sigma \nabla J\left(\bar{t}_{k}\right)^{\prime}\left(\bar{t}_{k}-\bar{t}_{k+1}\right),
$$

with $\sigma \in(0,1)$. The stepsize $s_{\mathrm{gp}, \mathrm{k}}$ satisfying (44) at iteration $k$ can be found by using backtracking, i.e., by examining for some fixed $\beta \in(0,1)$ and $s_{\text {init }}>0$ the sequence of values $\left\{s_{\text {init }}, \beta s_{\text {init }}, \beta^{2} s_{\text {init }}, \beta^{3} s_{\text {init }}, \ldots\right\}$ and taking as $s_{k}$ the largest one for which (44) holds.

Iteration of GP in (43) requires the gradient $\nabla J_{t o t}\left(\bar{t}_{k}\right)$ of the cost (38). To compute the gradient of the component $J_{\mathrm{c}}(\bar{t})$ at $\bar{t}$, it is necessary to perform a conversion $\bar{t} \rightarrow t_{I}$, i.e., to arrange the switching times in a non-decreasing order as explained in 3.4. Then for the computation of the partial derivative with respect to a switching time $t_{i j}$ in vector $\bar{t}$, we use its corresponding time $t_{I_{m}}$ in vector $t_{I}$. Since $\bar{t}$ and $t_{I}$ are essentially two vectors containing information about the same switching times, with slight abuse of notation in the sequel we will ocasionally be writing $J\left(t_{I}\right)$ instead of $J(\bar{t})$.

By using the chain rule, the partial derivative of the function $J_{\mathrm{c}}\left(t_{I}\right)$ in (33) with respect to $t_{I_{j}}, j \in\left\{1, \ldots, N_{s w}\right\}$, is given by

$$
\frac{\partial J_{\mathrm{c}}\left(t_{I}\right)}{\partial t_{I_{j}}}=\sum_{k=0}^{N_{S w}}\left(\frac{\partial \bar{x}_{k}^{\prime}}{\partial t_{I_{j}}} N_{k} \bar{x}_{k}+\bar{x}_{k}^{\prime} \frac{\partial N_{k}}{\partial t_{I_{j}}} \bar{x}_{k}+\bar{x}_{k}^{\prime} N_{k} \frac{\partial \bar{x}_{k}}{\partial t_{I_{j}}}\right),
$$

and it as well represents the partial derivative of $J_{c}(\bar{t})$ with respect to the coordinate of $\bar{t}$ corresponding to $t_{I_{j}}$.

The gradient expression (45) involves the partial derivatives $\partial \bar{x}_{k} / \partial t_{I_{j}}$ and $\partial N_{k} / \partial t_{I_{j}}$, whose expressions will be derived now. The expressions for the partial derivatives $\partial \bar{x}_{k} / \partial t_{I_{j}}$ are obtained from (31), and have the following form: 
- For $k>j+1$

$$
\begin{gathered}
\frac{\partial \bar{x}_{k}}{\partial t_{I_{j}}}=\prod_{m=j+1}^{k-1} e^{\bar{A}_{m}\left(t_{I_{m+1}}-t_{I_{m}}\right)}\left(-\bar{A}_{j}\right) \prod_{m=0}^{j} e^{\bar{A}_{m}\left(t_{I_{m+1}}-t_{I_{m}}\right)} \bar{x}_{0}+ \\
\prod_{m=j}^{k-1} e^{\bar{A}_{m}\left(t_{I_{m+1}}-t_{I_{m}}\right)} \bar{A}_{j-1} \prod_{m=0}^{j-1} e^{\bar{A}_{m}\left(t_{I_{m+1}}-t_{I_{m}}\right)} \bar{x}_{0} .
\end{gathered}
$$

- For $k=j+1$

$$
\begin{aligned}
\frac{\partial \bar{x}_{k}}{\partial t_{I_{j}}}= & \left(-\bar{A}_{j}\right) \prod_{m=0}^{j} e^{\bar{A}_{m}\left(t_{I_{m+1}}-t_{I_{m}}\right)} \bar{x}_{0}+ \\
& e^{\bar{A}_{j}\left(t_{I_{j+1}}-t_{I_{j}}\right)} \bar{A}_{j-1} \prod_{m=0}^{j-1} e^{\bar{A}_{m}\left(t_{I_{m+1}}-t_{I_{m}}\right)} \bar{x}_{0} .
\end{aligned}
$$

- For $k=j$

$$
\frac{\partial \bar{x}_{k}}{\partial t_{I_{j}}}=\bar{A}_{k-1} \prod_{m=0}^{k-1} e^{\bar{A}_{m}\left(t_{I_{m+1}}-t_{I_{m}}\right)} \bar{x}_{0} .
$$

- For $k<j$

$$
\frac{\partial \bar{x}_{k}}{\partial t_{I_{j}}}=0
$$

The expressions for the partial derivatives $\partial N_{k} / \partial t_{I_{j}}$ are obtained using (34)(36). By using (36) and denoting $m:=n+1$ where $n=17$ is the order of the system (22), the $N_{k}$ in (34) can be expressed as

$$
N_{k}=\left[\begin{array}{ll}
0 & I
\end{array}\right] e^{\hat{A}_{k}^{\prime}\left(t_{I_{k+1}}-t_{I_{k}}\right)}\left[\begin{array}{ll}
0 & 0 \\
I & 0
\end{array}\right] e^{\hat{A}_{k}\left(t_{I_{k+1}}-t_{I_{k}}\right)}\left[\begin{array}{l}
0 \\
I
\end{array}\right]
$$

where the $I$ and 0 denote $I_{m \times m}$ and $0_{m \times m}$, respectively. By introducing

$$
T_{1}:=\left[\begin{array}{ll}
0 & I
\end{array}\right], \quad T_{2}:=\left[\begin{array}{ll}
0 & 0 \\
I & 0
\end{array}\right], \quad T_{3}:=\left[\begin{array}{l}
0 \\
I
\end{array}\right],
$$

the partial derivatives of $N_{k}$ matrices are:

- For $k=j$

$$
\begin{aligned}
\frac{\partial N_{k}}{\partial t_{I_{j}}}= & T_{1} \cdot\left(-\hat{A}_{k}^{\prime}\right) \cdot e^{\hat{A}_{k}^{\prime}\left(t_{I_{k+1}}-t_{I_{k}}\right)} \cdot T_{2} \cdot e^{\hat{A}_{k}\left(t_{I_{k+1}}-t_{I_{k}}\right)} \cdot T_{3}+ \\
& T_{1} \cdot e^{\hat{A}_{k}^{\prime}\left(t_{I_{k+1}}-t_{I_{k}}\right)} \cdot T_{2} \cdot\left(-\hat{A}_{k}\right) \cdot e^{\hat{A}_{k}\left(t_{I_{k+1}}-t_{I_{k}}\right)} \cdot T_{3} .
\end{aligned}
$$


- For $k+1=j$

$$
\begin{array}{r}
\frac{\partial N_{k}}{\partial t_{I_{j}}}= \\
T_{1} \cdot \hat{A}_{k}^{\prime} \cdot e^{\hat{A}_{k}^{\prime}\left(t_{I_{k+1}}-t_{I_{k}}\right)} \cdot T_{2} \cdot e^{\hat{A}_{k}\left(t_{I_{k+1}}-t_{I_{k}}\right)} \cdot T_{3}+ \\
T_{1} \cdot e^{\hat{A}_{k}^{\prime}\left(t_{I_{k+1}}-t_{I_{k}}\right)} \cdot T_{2} \cdot \hat{A}_{k} \cdot e^{\hat{A}_{k}\left(t_{I_{k+1}}-t_{I_{k}}\right)} \cdot T_{3} .
\end{array}
$$

- For $k<j-1$ or $k>j$

$$
\frac{\partial N_{k}}{\partial t_{I_{j}}}=0_{m \times m}
$$

On the other hand, the partial derivative of the function $J_{\mathrm{d}}\left(t_{I}\right)$ in (38) with respect to $t_{I_{j}}, j \in\left\{1, \ldots, N_{s w}\right\}$, is given by

$$
\frac{\partial J_{\mathrm{d}}\left(t_{I}\right)}{\partial t_{I_{j}}}=2 q t_{I_{j}}
$$

An appropriate choice of the initial iterate $\bar{t}_{0} \in \mathbb{R}^{N_{s w}}$ for GP can be obtained by using the vector composed of the nominal switching times

$$
\bar{t}^{*}=\left[\begin{array}{lllllllll}
t_{\mathrm{a} 1}^{*} & \ldots & t_{\mathrm{aN}}^{*} & t_{\mathrm{b} 1}^{*} & \ldots & t_{\mathrm{bN}_{\mathrm{b}}}^{*} & t_{\mathrm{c} 1}^{*} & \ldots & t_{\mathrm{cN}_{\mathrm{c}}}^{*}
\end{array}\right]^{\prime} .
$$

which correspond to the employed OPP.

\subsection{Quadratic programming}

In order to obtain a quadratic approximation of the cost term $J_{\mathrm{c}}\left(t_{I}\right)$ in (33) with a possibility of reducing evaluations of the matrix exponentials online, the vectors $\bar{x}_{k}$ are linearized around the nominal switching times $t_{I}^{*}$ from OPP:

$$
\bar{x}_{k}\left(t_{I}\right) \approx \bar{x}_{k}\left(t_{I}^{*}\right)+\nabla \bar{x}_{k}\left(t_{I}^{*}\right) \Delta t_{I}, \quad \forall k \in\left\{0, \ldots, N_{s w}+1\right\},
$$

where $\Delta t_{I}=\left[\begin{array}{lll}\Delta t_{I_{1}} & \ldots & \Delta t_{I_{N_{s w}}}\end{array}\right]^{\prime}$ with elements $\Delta t_{I_{j}}=t_{I_{j}}-t_{I_{j}}^{*}, \forall j \in\left\{1, \ldots, N_{s w}\right\}$, and the Jacobian $\nabla \bar{x}_{k}\left(t_{I}^{*}\right)$ has the form

$$
\nabla \bar{x}_{k}\left(t_{I}^{*}\right)=\left[\begin{array}{lll}
\frac{\partial \bar{x}_{k}}{\partial t_{I_{1}}}\left(t_{I}^{*}\right) & \ldots & \frac{\partial \bar{x}_{k}}{\partial t_{I_{s w}}}\left(t_{I}^{*}\right)
\end{array}\right], \forall k \in\left\{0, \ldots, N_{s w}+1\right\},
$$

where the partial derivatives $\partial \bar{x}_{k} / \partial t_{I_{j}}$ are given in (46)-(49).

By introducing the linearizations (57) into the expression for $J_{\mathrm{c}}(33)$, its quadratic approximation is obtained:

$$
\begin{aligned}
J_{\mathrm{cQP}} & =\sum_{k=0}^{N_{s w}}\left(\bar{x}_{k}\left(t_{I}^{*}\right)+\nabla \bar{x}_{k}\left(t_{I}^{*}\right) \Delta t_{I}\right)^{\prime} \cdot N_{k} \cdot\left(\bar{x}_{k}\left(t_{I}^{*}\right)+\nabla \bar{x}_{k}\left(t_{I}^{*}\right) \Delta t_{I}\right) \\
& =\Delta t_{I}^{\prime} H_{Q P} \Delta t_{I}+h_{Q P}^{\prime} \Delta t_{I}+c_{Q P},
\end{aligned}
$$


where $H_{Q P}, h_{Q P}$ and $c_{Q P}$ are obtained by sorting the terms.

By taking into account as well the term $J_{\mathrm{d}}$, the quadratic approximation of the cost $J_{\text {tot }}$ in (38) is

$$
J_{\text {totQP }}=J_{\mathrm{cQP}}+\sum_{k=1}^{N_{S W}} q\left(\Delta t_{I_{k}}\right)^{2} .
$$

Since the vectors $\bar{x}_{k}$ are linearized around the nominal switching times $t_{I}^{*}$, the approximation $J_{\mathrm{cQP}}$ is valid only when the sequence of times $\bar{t}_{I}$ is kept unchanged from the sequence of $t_{I}^{*}$. This is imposed by introducing the sequence constraint $t_{0} \leq t_{I_{1}} \leq t_{I_{2}} \leq \cdots \leq t_{I_{N_{s w}-1}} \leq t_{I_{N_{s w}}} \leq t_{1}$, and by modifying it to also incorporate the minimal separation between the switching times $\delta$ we obtain the constraint

$$
t_{0} \leq t_{I_{1}}, t_{I_{1}}+\delta \leq t_{I_{2}}, \ldots, t_{I_{s w}}+\delta \leq t_{1} .
$$

where $t_{I_{j}}=t_{I_{j}}^{*}+\Delta t_{I_{j}}, \forall j \in\left\{1, \ldots, N_{s w}\right\}$, as previously defined. Notice that this sequence constraint is a subset of the constraint (40)-(42), which thus can be removed.

Finally, since the linearizations (57) are valid only for small values of deviations $\Delta t_{I}$, a box constraint is imposed as

$$
l_{b} \leq \Delta t_{I_{k}} \leq u_{b}, \quad \forall k \in\left\{1,2, \ldots, N_{s w}\right\}
$$

In summary, the optimization problem with quadratic approximation of the cost involves the quadratic cost function (59) and the constraint set composed of sequence and box constraint (60) and (61), respectively. It can be noticed that the constraint set is a polytope.

\subsection{Additional aspects of GP and QP approach}

The GP approach requires evaluations of matrix exponentials. Since the number of voltage levels $\mathscr{L}$ a power converter can supply is finite (equal to $N_{\text {lvl }}$ ), the corresponding number of $\bar{A}$ and $\hat{A}$ matrices is finite as well. Thus, one could facilitate the evaluation of matrix exponentials by storing the offline computed matrices $e^{\bar{A}_{m} \tau}$ and $e^{\hat{A}_{m} \tau}$ evaluated for values of the gridded interval $\left[\tau_{\min }, \tau_{\max }\right]$. The simulation experiments show that a relatively small number of GP iterations, about 10, provides a sufficient computational power for even the most demanding transients. It should also be noted that application of GP requires continuous differentiability of the cost function, which is in our case not the case due to the discontinuities obtained when two switching times in $t_{I}$ switch their order. However, during computational experiments there were not detected any negative consequences of this discontinuity. 
Table 1: Converter, $L C L$ filter and grid parameters.

\begin{tabular}{|l|lr|}
\hline Parameter & & Value \\
\hline \hline Converter's number of levels & $N_{\mathrm{lvl}}$ & 3 \\
Converter's DC link voltage & $v_{\mathrm{dc}}$ & $5200 \mathrm{~V}$ \\
LCL inverter-side inductance & $L_{\mathrm{fi}}$ & $600 \mu \mathrm{H}$ \\
LCL inverter-side resistance & $R_{\mathrm{fi}}$ & $5 \mathrm{~m} \Omega$ \\
LCL capacitance & $C_{\mathrm{f}}$ & $1 \mathrm{mF}$ \\
LCL grid-side inductance & $L_{\mathrm{fg}}$ & $600 \mu \mathrm{H}$ \\
LCL grid-side resistance & $R_{\mathrm{fg}}$ & $5 \mathrm{~m} \Omega$ \\
Grid voltage & $V_{\mathrm{g}}$ & $3000 \mathrm{~V}$ \\
Grid nominal current & $I_{\mathrm{g}, \mathrm{n}}$ & $1540 \mathrm{~A}$ \\
Grid frequency & $f_{\mathrm{g}}$ & $50 \mathrm{~Hz}$ \\
\hline
\end{tabular}

The QP solution approach requires matrix exponentials $e^{\bar{A}_{m} \tau}$ and $e^{\hat{A}_{m} \tau}$ computed only for $\tau$ being the differences of the nominal switching times, plus the case in which the $\tau$ involves $t_{0}$, i.e., $\tau=t_{I_{1}}-t_{I_{0}}$. In all performed simulation tests of the described method, the quadratic cost function was never detected to be non-convex.

\section{Simulation Results}

The performance of the developed controller in steady-state and transients is explored by Matlab simulation, examining both the GP and QP solution approach.

\subsection{System and controller parameters}

The system parameters are taken from [15] and are given in Table 1. The controller parameters are given in Table 2.

\subsection{Steady-state performance of GP and QP approach}

The steady-state performance is measured by using total harmonic distortion, as defined in (25). Since the expression for the THD can be applied to each one of the three phases, the average of the THD in each of the three phases is considered. The steady-state simulations have been run for a range of referent apparent powers to be injected to the grid of the form

$$
S_{g}=\kappa\left(P_{g, n o m}+\mathrm{j} \mathrm{Q}_{\mathrm{g}, \text { nom }}\right),
$$


Table 2: Controller parameters for GP and QP approach.

\begin{tabular}{|l|lr|}
\hline Parameter & & Value \\
\hline \hline Controller's sampling time & $T_{\mathrm{s}}$ & $25 \mu \mathrm{s}$ \\
Switches in prediction horizon & $N_{\mathrm{sw}}$ & 9 \\
Minimal separation of switches & $\delta$ & $27.5 \mu \mathrm{s}$ \\
OPP number of pulses & $d$ & 8 \\
Penalties in $Q$ for inverter current & $Q_{\mathrm{fi}}$ & 0 \\
Penalties in $Q$ for grid current & $Q_{\mathrm{fg}}$ & 100 \\
Penalties in $Q$ for capacitor voltage & $Q_{\mathrm{f}}$ & 10 \\
Penalties on deviations from nominal times & $q$ & 100 \\
GP's number of gradient iterations & $N_{\mathrm{GP}}$ & 10 \\
GP's sufficient cost decrease coefficient & $\sigma$ & $5 \cdot 10^{-3}$ \\
GP's backtracking coefficient & $\beta$ & 0.3 \\
GP's initial backtracking stepsize & $s_{\mathrm{init}}$ & $1 \cdot 10^{-11}$ \\
QP's lower bound of box constraint & $l_{b}$ & $-50 \mu \mathrm{s}$ \\
QP's upper bound of box constraint & $u_{b}$ & $50 \mu \mathrm{s}$ \\
\hline
\end{tabular}

where the paramater $\kappa$ is varied from -1 to +1 in steps of 0.2 , and we select $P_{g, \text { nom }}=Q_{g, \text { nom }}=8 \mathrm{MVA}$. The values of the THD obtained with GP and QP approach are given in Table 3 and represented in Fig 3. It can be noticed that the QP approach can cause a reduction of steady-state performance which is at some operating points more significant than on the others. 
Table 3: Steady-state THD values with GP and QP.

\begin{tabular}{|c||c|c|}
\hline$\kappa$ & GP THD [\%] & QP THD [\%] \\
\hline \hline 1 & 0.14 & 0.27 \\
\hline 0.8 & 0.20 & 0.40 \\
\hline 0.6 & 0.30 & 0.28 \\
\hline 0.4 & 0.58 & 0.72 \\
\hline 0.2 & 1.26 & 1.25 \\
\hline-0.2 & 0.67 & 0.61 \\
\hline-0.4 & 0.45 & 1.65 \\
\hline-0.6 & 0.28 & 0.46 \\
\hline-0.8 & 0.24 & 2.42 \\
\hline-1 & 0.13 & 0.13 \\
\hline
\end{tabular}

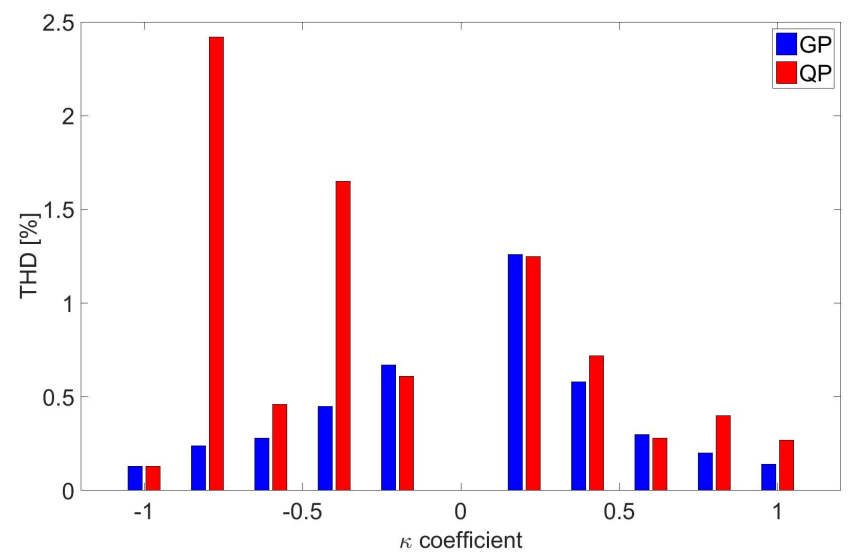

Figure 3: Steady-state THD values obtained with GP and QP.

\subsection{Transient performance of GP and QP approach}

The transient performance is tested by applying step changes of reference injection power. The reference power is again specified as in (62), and a step change is introduced by the value of $\kappa$. The simulation results obtained by changing $\kappa=1$ to values 0.6 and 0 are given on Fig. 4 . It can be noticed from simulation results that for step changes of smaller sizes, the GP and QP solution approach give similar transient behaviours. For larger step changes, the QP approach has 
worse performance since the corrections $\Delta t_{I}$ that it can apply are limited by the box constraint.

\section{Conclusion}

In this paper we have developed an MPC scheme for power electronics application which employs integral squared tracking error as its cost function and combines it with OPPs. The combination of these two ingredients results in a controller characterized by a great transient behaviour and steady-state performance. The obtained optimization problem is approached by using GP algorithm and by linearizing the cost function which brought the problem the form of QP. The performance of the controller is examined by Matlab simulations. The future work will further investigate the solution possibilities for the optimization problem involved in the controller.

\section{References}

[1] W. Leonhard, Control of electrical drives. Springer Science \& Business Media, 2001.

[2] T. Geyer, G. Papafotiou, and M. Morari, "Model predictive direct torque control-part i: Concept, algorithm, and analysis," IEEE Transactions on Industrial Electronics, vol. 56, no. 6, pp. 1894-1905, June 2009.

[3] T. Geyer, N. Oikonomou, G. Papafotiou, and F. D. Kieferndorf, "Model predictive pulse pattern control," IEEE Transactions on Industry Applications, vol. 48, no. 2, pp. 663-676, March 2012.

[4] T. Geyer, "Generalized model predictive direct torque control: Long prediction horizons and minimization of switching losses," in Decision and Control, 2009 held jointly with the 2009 28th Chinese Control Conference. CDC/CCC 2009. Proceedings of the 48th IEEE Conference on, Dec 2009, pp. 6799-6804.

[5] — - "Model predictive direct current control: Formulation of the stator current bounds and the concept of the switching horizon," IEEE Industry Applications Magazine, vol. 18, no. 2, pp. 47-59, March 2012.

[6] G. S. Buja, "Optimum output waveforms in pwm inverters," IEEE Transactions on Industry Applications, vol. IA-16, no. 6, pp. 830-836, Nov 1980. 

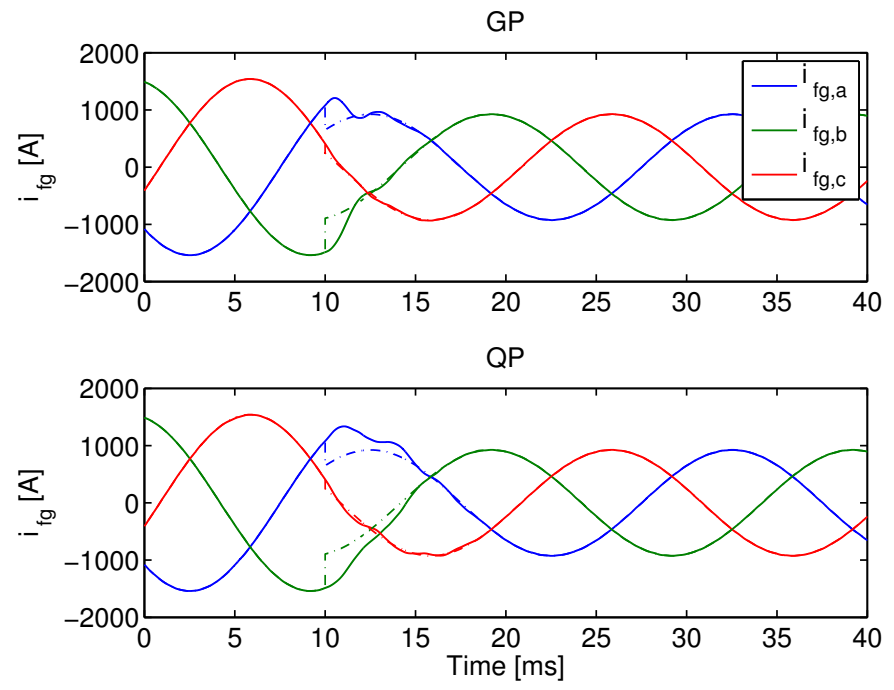

(a) Transient for $\kappa=1$ to $\kappa=0.6$.
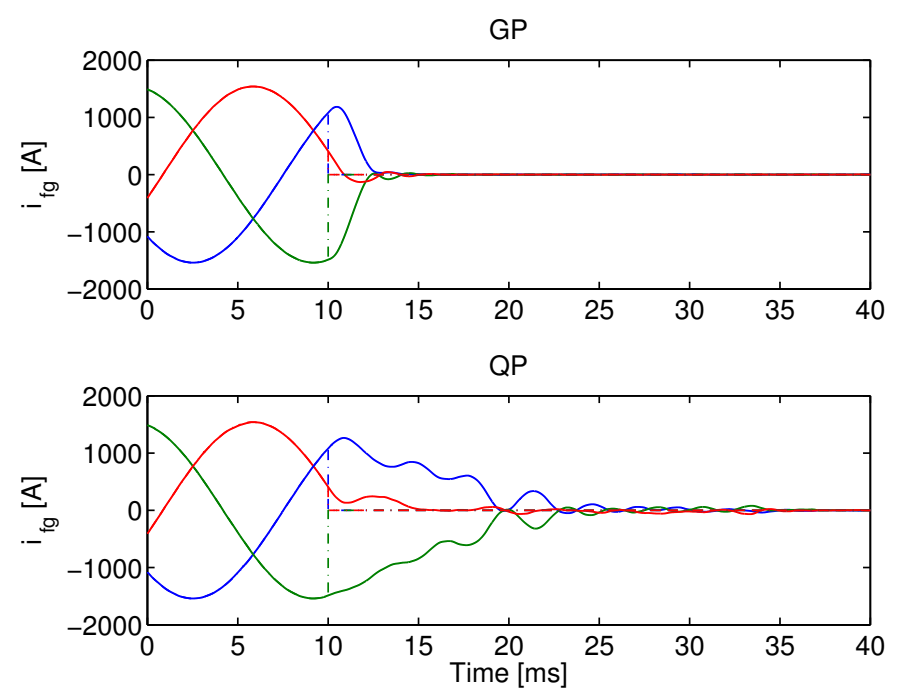

(b) Transient for $\kappa=1$ to $\kappa=0$.

Figure 4: Transients of grid current $i_{\mathrm{fg}}$ with GP and QP, obtained by changing at $t=10 \mathrm{~ms}$ the parameter $\kappa=1$ in equation (62) to the values (a) $\kappa=0.6$, (b) $\kappa=0$. 
[7] A. K. Rathore, J. Holtz, and T. Boller, "Synchronous optimal pulsewidth modulation for low-switching-frequency control of medium-voltage multilevel inverters," IEEE Transactions on Industrial Electronics, vol. 57, no. 7, pp. 2374-2381, July 2010.

[8] S. Almér, S. Mariéthoz, and M. Morari, "Sampled Data Model Predictive Control of a Voltage Source Inverter for Reduced Harmonic Distortion," IEEE Transactions on Control Systems Technology, vol. 21, no. 5, pp. 19071915, Sept 2013.

[9] —_, "Dynamic Phasor Model Predictive Control of Switched Mode Power Converters," IEEE Transactions on Control Systems Technology, vol. 23, no. 1, pp. 349-356, Jan 2015.

[10] S. Almér, "Model Predictive PWM of a Single Phase Inverter: A Nonlinear Transformation Approach," in Energy Conversion Congress and Exposition (ECCE), 2015 IEEE, Sept 2015, pp. 4301-4306.

[11] E. Clarke, Circuit analysis of AC power systems. Wiley, 1943, vol. 1.

[12] J. G. Kassakian, M. F. Schlecht, and G. C. Verghese, Principles of power electronics. Graphis, 2000.

[13] C. V. Loan, "Computing integrals involving the matrix exponential," IEEE Transactions on Automatic Control, vol. 23, no. 3, pp. 395-404, Jun 1978.

[14] D. P. Bertsekas, Nonlinear Programming: 2nd Edition. Athena Scientific, 2004.

[15] J. Scoltock, T. Geyer, and U. Madawala, "Model predictive direct power control for a grid-connected converter with an lcl-filter," in Industrial Technology (ICIT), 2013 IEEE International Conference on, Feb 2013, pp. 588593. 